\title{
BMJ Open Screening for seemingly healthy newborns with congenital cytomegalovirus infection by quantitative real-time polymerase chain reaction using newborn urine: an observational study
}

\author{
Akira Yamaguchi, ${ }^{1,2}$ Tsutomu Oh-ishi, ${ }^{3}$ Takashi Arai, ${ }^{1,2}$ Hideaki Sakata, ${ }^{4,5}$ \\ Nodoka Adachi, ${ }^{6}$ Satoshi Asanuma, ${ }^{6}$ Eiji Oguma, ${ }^{7}$ Hirofumi Kimoto, ${ }^{8}$ \\ Jiro Matsumoto, ${ }^{9}$ Hidetoshi Fujita, ${ }^{10}$ Tadashi Uesato, ${ }^{10}$ Jutaro Fujita, ${ }^{10}$ \\ Ken Shirato, ${ }^{11}$ Hideki Ohno, ${ }^{11}$ Takako Kizaki ${ }^{11}$
}

To cite: Yamaguchi A, Ohishi T, Arai T, et al. Screening for seemingly healthy newborns with congenital cytomegalovirus infection by quantitative real-time polymerase chain reaction using newborn urine: an observational study. BMJ Open 2017;7:e013810. doi:10.1136/bmjopen-2016013810

- Prepublication history for this paper is available online. To view these files please visit the journal online (http://dx.doi.org/10.1136/ bmjopen-2016-013810).

Received 23 August 2016 Revised 29 December 2016 Accepted 30 December 2016

CrossMark

For numbered affiliations see end of article.

Correspondence to Dr Takako Kizaki; kizaki@ks.kyorin-u.ac.jp

\section{ABSTRACT}

Objective: Approximately $8-10 \%$ of newborns with asymptomatic congenital cytomegalovirus (cCMV) infection develop sensorineural hearing loss (SNHL). However, the relationship between CMV load, SNHL and central nervous system (CNS) damage in CCMV infection remains unclear. This study aimed to examine the relationship between urinary CMV load, SNHL and CNS damage in newborns with CCMV infection.

Study design: The study included 23368 newborns from two maternity hospitals in Saitama Prefecture, Japan. Urine screening for CCMV infection (quantitative real-time PCR) and newborn hearing screening (automated auditory brainstem response (AABR) testing) were conducted within 5 days of birth to examine the incidence of CCMV infection and SNHL, respectively. CNS damage was assessed by MRI of cCMV-infected newborns.

Results: The incidence of cCMV infection was 60/ $23368(0.257 \%$; $95 \% \mathrm{Cl} 0.192 \%$ to $0.322 \%)$. The geometric mean urinary CMV DNA copy number in newborns with CCMV was $1.79 \times 10^{6}$ copies $/ \mathrm{mL}(95 \%$ CI $7.97 \times 10^{5}$ to $\left.4.02 \times 10^{6}\right)$. AABR testing revealed abnormalities in 171 of the $22229(0.769 \%)$ newborns whose parents approved hearing screening. Of these 171 newborns, 22 had SNHL (12.9\%), and 5 of these 22 were infected with CCMV (22.7\%). Newborns with both CCMV and SNHL had a higher urinary CMV DNA copy number than newborns with CCMV without SNHL $(p=0.036)$. MRI revealed CNS damage, including white matter abnormalities, in $83.0 \%$ of newborns with cCMV. Moreover, newborns with CNS damage had a significantly greater urinary CMV load than newborns without CNS damage ( $p=0.013$ ).

Conclusions: We determined the incidence of $\mathrm{CCMV}$ infection and urinary CMV DNA copy number in seemingly healthy newborns from two hospitals in

\section{Strengths and limitations of this study}

- Verification of the risk factors for sensorineural hearing loss (SNHL) in newborns and late-onset SNHL caused by congenital cytomegalovirus (cCMV) infection as well as for developmental or intellectual disability is important for predicting a patient's prognosis. In this study we examined the association of urinary CMV load in CCMV infection cases with SNHL and central nervous system (CNS) damage.

- Urinary CMV load was evaluated by calculating the CMV DNA copy number in a urine sample by using quantitative real-time PCR ( $(\mathrm{PCR}$ ) while ensuring sensitivity and quantitative capability.

- The CMV DNA copy number of newborns with SNHL and CNS damage was significantly more than that of newborns without any findings. This suggests that measuring CMV load in urine samples could be useful for predicting prognosis of CCMV infection.

- Based on the current research, it is unclear whether CNS damage related to CCMV infection is associated with future developmental or intellectual disability, which indicates that follow-up examinations that include intelligence and psychological evaluation are required.

- The use of qPCR for a urine sample as a screening test requires improvements in usability and economic efficiency.

Saitama Prefecture. SNHL and CNS damage were associated with urinary CMV DNA copy number. Quantification of urinary CMV load may effectively predict the incidence of late-onset SNHL and neurodevelopmental disorders. 


\section{INTRODUCTION}

Human cytomegalovirus (CMV) is known to cause congenital infection and is the most commonly implicated pathogen in the group of congenital infections known as TORCH syndrome (toxoplasmosis, rubella, CMV infection, herpes simplex and other infections). The incidence of $\mathrm{CMV}$ is $0.2-2.4 \%$ in all newborns, and approximately $5-10 \%$ of CMV-infected newborns have symptomatic congenital CMV (cCMV) infection. In cases of severe infection, newborns can present with symptoms of cytomegalic inclusion disease (intrauterine growth retardation, hepatosplenomegaly, jaundice, blueberry muffin rash, thrombocytopenia, purpura, microcephaly, intracranial calcification and developmental delay). In contrast, cCMV infection is asymptomatic in 90-95\% of infected newborns. ${ }^{1}$

In the USA the incidence of perinatal sensorineural hearing loss (SNHL) is estimated to be $0.186 \%$, and the prevalence of hearing loss (HL) among 4-year-olds is estimated to be $0.27 \%$. Twenty-one per cent of cases of perinatal SNHL and 25\% of cases of HL among 4-year-olds are estimated to be caused by cCMV infection. ${ }^{2}$ Fowler $e t a \hat{l}^{3}$ reported that $7.2 \%$ of children with asymptomatic cCMV infection developed congenital SNHL and that $18.2 \%$ of asymptomatic children with HL developed late-onset SNHL. Thus, the high incidence of SNHL in 4-year-olds could be attributed to late-onset SNHL, occurring as a result of cCMV infection. However, newborn hearing screening cannot predict or identify late-onset SNHL due to asymptomatic cCMV infection. ${ }^{4}$

CMV infection of the central nervous system (CNS) presents as encephalitis and periependymitis, and can cause ventriculomegaly, gliosis and calcification once encephalitis resolves. CMV infection is also known to cause developmental delay and seizures. MRI has been used to show the CNS damage associated with cCMV infection, ${ }^{5}{ }^{6}$ but the relationship between CMV load and SNHL and CNS damage has not yet been fully ascertained.

Several techniques have been used to screen for CMV infection in newborns. Numazaki et $a l^{7}$ used traditional culturing of CMV from urine to determine the incidence of cCMV infection in Sapporo, Japan. In another multicentre study conducted in Japan, Koyano et al used FTA-Elute filter cards (GE Healthcare Life Sciences, Piscataway, New Jersey, USA) placed in the diapers of newborns to collect urine and then dried the cards and subjected them to quantitative real-time PCR (qPCR) to determine the incidence of cCMV infection. Isolating CMV from urine represents a reliable screening technique for CMV infection, but involves a complicated and time-consuming procedure and the result may not be ascertained for up to 2 weeks. In contrast, the use of FTA-Elute filter cards is easier, economical and forceful, but the correlation between urinary CMV loads, sequelae and MRI findings of neurological damage is equivocal in the newborn screening for cCMV infection. The accurate determination of CMV loads in urine using qPCR may therefore be a good tool to assess newborns with a risk of sequelae, and could be especially helpful in determining the need for antiviral treatment.

In this study, DNA was extracted from liquid urine collected within 5 days of birth and was subjected to qPCR to screen for cCMV infection. Subsequently, the association between the screening results and incidence of SNHL was examined. This study also used MRI to investigate CNS damage in newborns with cCMV.

\section{METHODS}

\section{Subjects and initial testing}

The subjects were newborn infants from two maternity hospitals in Saitama Prefecture (Aiwa Hospital, Kawagoe and Sannoh Clinic, Shiraoka, Saitama) and were recruited between 2 December 2008 and 31 May 2015. The mothers of 23368 newborns agreed to participate in this study. Of these 23368 newborns, 22229 (95.1\%) underwent newborn hearing screening using automated auditory brainstem response (AABR) testing.

Head circumference (HC) at birth was measured in addition to weight and height and was compared with that addressed under the 2010 Japan Child Growth Standards (Ministry of Health, Labour and Welfare). HC $\mathrm{z}$ score modified by Noyola $e t a t^{\vartheta}$ was calculated, and HC $\mathrm{z}$ score $<-2$ was considered microcephaly.

\section{Hearing screening}

If the hearing screening revealed abnormalities, the newborn underwent more precise testing of the auditory brainstem response (ABR), auditory steady-state responses and otoacoustic emissions at the Mejiro University Audiology Clinic. When a newborn was diagnosed with SNHL based on this precise testing, the gap junction protein $\beta 2$ (GJB2) or connexin 26 gene was sequenced to identify mutations. Mutations in these genes are responsible for roughly $20 \%$ of SNHL cases. ${ }^{10}$

\section{Urine collection and testing}

Urine was collected in a urine collection bag 0-5 days after birth and then transferred to a sterile plastic urine collection tube. Urine was then frozen at $-20^{\circ} \mathrm{C}$ and transported to Saitama Children's Medical Center, where DNA was extracted within 10 days. Newborns in whose urine CMV was detected underwent MRI at Saitama Children's Medical Center to identify CNS damage. The CMV load in their urine was then retested.

\section{DNA extraction and qPCR}

A QIAamp DNA Blood Mini Kit (Qiagen, Hilden, Germany) was used to extract DNA from urine samples in accordance with the manufacturer's instructions. ${ }^{11}$ CMV DNA was subjected to qPCR to detect the pp65 gene (GenBank: accession number NC_006273), as previously described by Griscelli et al. ${ }^{12}$ Primers and a fluorescence-labelled probe were added to the reaction 
mixture (TaqMan Real-Time qPCR Master Mix, Life Technologies, Carlsbad, California, USA) and CMV DNA was amplified. The PCR products were quantified using an ABI 7900 HT FAST Real-time PCR System (ABI, Foster City, California, USA). The sensitivity of qPCR detection was $\geq 100$ copies $/ \mathrm{mL}$.

\section{Identification of mutations in GJB2}

DNA samples were extracted from peripheral blood mononuclear cells using a QIAamp DNA Blood Mini Kit (Qiagen) and the GJB2 gene was directly sequenced as previously described. ${ }^{13}$

\section{Identification of CNS damage using MRI}

Head MRI was performed using a $1.5 \mathrm{~T}$ MRI scanner (Philips, MRI Systems Achieva 1.5T, Amsterdam, The Netherlands) and the images were analysed by two paediatric radiologists with over 15 years of experience. CNS damage was identified in images acquired using T1-weighted spin-echo (SE) sequences in the sagittal and axial planes and a T2-weighted fast $\mathrm{SE}$ sequence in the axial or coronal plane. CNS damage was assessed on the basis of a combination of criteria for white matter (signal abnormality, loss of volume, presence of a cyst, ventriculomegaly, corpus callosal thinning or myelination) and grey matter (grey matter signal abnormality, gyration or presence of subarachnoid spaces). In some instances, a $\mathrm{T} 2 *$-weighted or T1-weighted inversion recovery (IR) sequence was added to accurately assess calcification of soft tissue or myelin abnormalities. Myelination is thought to be mostly completed by 18 months when assessed using a fluid-attenuated IR sequence. White matter abnormalities (WMAs) were assessed on T2-weighted images, ${ }^{5} 14{ }^{15}$ essentially using the criteria defined by van der Voorn et al. ${ }^{16}$ The criteria for patients with cerebral WMAs only were as follows: (1) multifocal white matter lesions were present; (2) the lesions were predominantly located in the deep white matter; and (3) the largest lesions were located in the parietal region. The criteria for patients with both cerebral white matter and gyral abnormalities were as follows: (1) dysgyria and MRI findings suggestive of polymicrogyria were present; and (2) either multifocal WMAs (like those described for the patients with cerebral WMAs only) or diffuse WMAs were present.

CT was performed on newborns with suspected calcification revealed by MRI.

\section{Statistical analysis}

Statistical analyses were performed using SPSS V.19.0 (IBM, Tokyo, Japan). The number of copies of CMV was expressed as a geometric mean (GM) $(95 \%$ CI). Data were compared using a two-sided non-parametric Mann-Whitney $\mathrm{U}$ test, and $\mathrm{p}<0.05$ was considered to represent statistical significance. Mother's age, gestational age, newborn's birth weight and $\mathrm{HC}$ at birth were expressed as an arithmetic mean (AM) \pm 2 SE. Pearson's $\chi^{2}$ test was used for testing the proportion.

\section{Ethical considerations}

This study was fully explained to the parents of the newborns before obtaining parental consent, and was approved by the ethics committee of Saitama Children's Medical Center.

\section{RESULTS}

\section{General characteristics of the screened newborns}

Sixty of the 23368 newborns tested positive for cCMV $(0.257 \%$; $95 \%$ CI $0.192 \%$ to $0.322 \%)$. At birth, newborns with cCMV infection weighed $2895 \pm 96 \mathrm{~g}(\mathrm{AM} \pm 2 \mathrm{SE})$, had a $\mathrm{HC}$ of $32.8 \pm 0.5 \mathrm{~cm}$ and a gestational age of 39.0 \pm 0.4 weeks. At the birth of newborns with cCMV infection the mothers were aged $30.2 \pm 1.5$ years. The urine of newborns with cCMV infection contained $1.79 \times 10^{6}$ copies $/ \mathrm{mL}$ CMV DNA (GM) $\left(95 \%\right.$ CI $7.97 \times 10^{5}$ to $4.02 \times 10^{6}$ copies $/ \mathrm{mL}$ ). No CMV DNA was detected in the urine of the other 23308 .

\section{Clinical manifestations of cCMV infection}

The clinical manifestations of cCMV in 60 newborns are shown in table 1 . The first or second MRI examination and CT revealed CNS damage in 44 newborns (figures 1-3). Calcification was noted in one newborn, polymicrogyria in one, ventriculomegaly in four, a periventricular cyst in two, hippocampal dysplasia in one and WMAs in all 44, and some newborns had multiple abnormal manifestations. In addition, microcephaly was noted in one newborn with a modified $\mathrm{HC}$ z score of -2.95 . SNHL was noted in five newborns and hemiplegia in one. At birth, all newborns appeared normal without any signs of cCMV infection aside from microcephaly. One newborn with microcephaly, however, did not present with any other features of symptomatic cCMV infection and thus was not suspected of having cCMV infection before her urine screen tested positive for CMV.

\section{Newborn hearing screening}

A total of 22229 newborns underwent AABR testing, which revealed abnormalities in $171(0.769 \%)$. More precise testing revealed abnormalities in 22 of these 171 $(12.9 \%)$, and these 22 newborns were thus diagnosed with SNHL. Of the 22 newborns diagnosed with SNHL, five $(22.7 \%)$ were infected with cCMV. All 22 newborns with SNHL underwent genetic testing to detect GJB2 mutations, but no newborns had both GJB2 genetic mutations and cCMV infection. Of the 60 newborns infected with cCMV, however, seven did not undergo AABR testing.

\section{Relationship between cCMV and SNHL}

The urinary CMV DNA copy number in five newborns with SNHL $\left(3.23 \times 10^{7}\right.$ copies $/ \mathrm{mL}, 95 \%$ CI $3.02 \times 10^{6}$ to $3.45 \times 10^{8}$ copies $/ \mathrm{mL}$ ) was significantly higher than that in the 48 newborns with cCMV without SNHL $\left(1.65 \times 10^{6}\right.$ copies $/ \mathrm{mL}, 95 \%$ CI $7.02 \times 10^{5}$ to $3.86 \times 10^{6}$ copies $/ \mathrm{mL}$; 
Table 1 Characteristics of patients with congenital cytomegalovirus (cCMV) infection

\begin{tabular}{|c|c|c|c|c|c|c|c|c|c|c|c|}
\hline \multirow[b]{2}{*}{$\begin{array}{l}\text { Pt } \\
\text { no }\end{array}$} & \multicolumn{2}{|c|}{$\begin{array}{l}\text { Screening for } \\
\text { CMV }\end{array}$} & \multicolumn{2}{|c|}{$\begin{array}{l}\text { Re-examination of } \\
\text { CMV }\end{array}$} & \multicolumn{2}{|c|}{ First MRI examination } & \multicolumn{2}{|c|}{ Second MRI examination } & \multicolumn{2}{|l|}{ AABR } & \multirow[b]{2}{*}{ Outcome* $^{*}$} \\
\hline & $\begin{array}{l}\text { Age } \\
\text { (days) }\end{array}$ & $\begin{array}{l}\text { Copies/ } \\
\mathrm{mL}\end{array}$ & $\begin{array}{l}\text { Age } \\
\text { (weeks) }\end{array}$ & $\begin{array}{l}\text { Copies/ } \\
\mathrm{mL}\end{array}$ & $\begin{array}{l}\text { Age } \\
\text { (weeks) }\end{array}$ & Findings & $\begin{array}{l}\text { Age } \\
\text { (months) }\end{array}$ & Findings & Right & Left & \\
\hline $1 \dagger$ & 1 & $1.9 \times 10^{8}$ & 5 & $1.9 \times 10^{8}$ & 6 & WMA, periventricular cysts & 27 & WMA, ventriculomegaly & $\begin{array}{l}R \\
(-90 \mathrm{~dB}) \ddagger\end{array}$ & $\mathrm{P}$ & $\begin{array}{l}\text { SNHL, PDD, speech } \\
\text { delay }\end{array}$ \\
\hline $2 \dagger$ & 4 & $8.0 \times 10^{6}$ & 2 & $7.2 \times 10^{7}$ & 12 & WMA & 18 & WMA & $\begin{array}{l}R \\
(-100 \mathrm{~dB})\end{array}$ & $R(-70 \mathrm{~dB})$ & $\begin{array}{l}\text { SNHL, PDD, speech } \\
\text { delay, calcification§ }\end{array}$ \\
\hline $3 \dagger$ & 0 & $3.2 \times 10^{8}$ & 24 & $2.5 \times 10^{6}$ & 78 & $\begin{array}{l}\text { WMA, ventriculomegaly, } \\
\text { hippocampal dysplasia }\end{array}$ & Declined & & $(-80 \mathrm{~dB})$ & $\begin{array}{l}\mathrm{R} \text { (no } \\
\text { response) }\end{array}$ & $\begin{array}{l}\text { SNHL, PDD, speech } \\
\text { delay }\end{array}$ \\
\hline $4 \dagger$ & 4 & $4.8 \times 10^{6}$ & 3 & $7.1 \times 10^{7}$ & 4 & WMA & 18 & WMA & $\begin{array}{l}R \\
(-100 \mathrm{~dB})\end{array}$ & $\mathrm{P}$ & $\begin{array}{l}\text { SNHL, PDD, speech } \\
\text { delay }\end{array}$ \\
\hline $5 \dagger$ & 1 & $1.5 \times 10^{7}$ & 32 & $2.1 \times 10^{7}$ & 6 & WMA & 9 & $\begin{array}{l}\text { WMA, cerebral parenchymal } \\
\text { atrophy }\end{array}$ & ND & ND & $\begin{array}{l}\text { Late-onset hearing } \\
\text { loss }\end{array}$ \\
\hline 6 & 1 & $4.1 \times 10^{6}$ & 10 & $1.8 \times 10^{6}$ & 12 & $\begin{array}{l}\text { Polymicrogyria, ventriculomegaly, } \\
\text { WMA }\end{array}$ & 18 & $\begin{array}{l}\text { Polymicrogyria, WMA, } \\
\text { ventriculomegaly, } \\
\text { periventricular cysts }\end{array}$ & $\mathrm{P}$ & $P$ & $\begin{array}{l}\text { Left hemiparesis, } \\
\text { PDD }\end{array}$ \\
\hline 7 & 2 & $1.0 \times 10^{7}$ & 5 & $1.8 \times 10^{8}$ & 8 & WMA & 18 & WMA & $\mathrm{P}$ & $P$ & $\begin{array}{l}\text { Microcephaly, } \\
\text { speech delay }\end{array}$ \\
\hline 8 & 4 & $5.2 \times 10^{5}$ & 9 & $2.0 \times 10^{6}$ & 7 & $\begin{array}{l}\text { WMA, vestibulo-semicircular canal } \\
\text { dysplasia }\end{array}$ & \multicolumn{2}{|c|}{ Not yet doneף } & $\mathrm{P}$ & $P$ & \\
\hline 9 & 1 & $8.0 \times 10^{6}$ & 41 & $2.5 \times 10^{7}$ & 40 & WMA, infarct & 18 & WMA & $P$ & $P$ & \\
\hline 10 & 4 & $2.1 \times 10^{6}$ & 6 & $8.6 \times 10^{7}$ & 7 & $\begin{array}{l}\text { WMA, coagulation, necrosis } \\
\text { around posterior horn of both } \\
\text { lateral ventricles }\end{array}$ & 18 & WMA & $P$ & $P$ & \\
\hline 11 & 1 & $2.5 \times 10^{6}$ & 5 & $5.3 \times 10^{7}$ & 5 & WMA & 18 & WMA, ventriculomegaly & $P$ & $P$ & \\
\hline 12 & 1 & $3.1 \times 10^{7}$ & 10 & $3.1 \times 10^{7}$ & 12 & WMA & 18 & WMA & $P$ & $\mathrm{P}$ & \\
\hline 13 & 2 & $2.0 \times 10^{5}$ & 6 & $5.0 \times 10^{4}$ & 11 & WMA & 18 & WMA & $\mathrm{P}$ & $P$ & \\
\hline 14 & 4 & $3.3 \times 10^{8}$ & 6 & $4.9 \times 10^{7}$ & 6 & WMA & 18 & WMA & $\mathrm{P}$ & $\mathrm{P}$ & \\
\hline 15 & 4 & $5.4 \times 10^{8}$ & 8 & $4.3 \times 10^{8}$ & 6 & WMA & 18 & WMA & $\mathrm{P}$ & $\mathrm{P}$ & \\
\hline 16 & 4 & $1.3 \times 10^{5}$ & 8 & $2.2 \times 10^{6}$ & 11 & WMA & 18 & WMA & $P$ & $P$ & \\
\hline 17 & 4 & $1.0 \times 10^{7}$ & 5 & $3.8 \times 10^{6}$ & 7 & WMA & 18 & WMA & $P$ & $P$ & \\
\hline 18 & 3 & $1.3 \times 10^{7}$ & 4 & $2.3 \times 10^{6}$ & 12 & WMA & 19 & WMA & $P$ & $P$ & \\
\hline 19 & 4 & $5.7 \times 10^{6}$ & 3 & $4.5 \times 10^{6}$ & 3 & WMA & 9 & WMA & $P$ & $P$ & \\
\hline 20 & 4 & $2.3 \times 10^{5}$ & \multicolumn{2}{|c|}{ No urine collection } & 8 & WMA & 12 & WMA & $\mathrm{P}$ & $\mathrm{P}$ & \\
\hline 21 & 1 & $4.8 \times 10^{7}$ & 5 & $9.4 \times 10^{5}$ & 8 & WMA & 11 & WMA & $\mathrm{P}$ & $\mathrm{P}$ & \\
\hline 22 & 4 & $4.8 \times 10^{7}$ & 5 & $1.1 \times 10^{7}$ & 6 & WMA & 17 & WMA & ND & ND & \\
\hline 23 & 4 & $1.4 \times 10^{5}$ & 10 & $1.4 \times 10^{7}$ & 12 & WMA & 18 & WMA & P & P & \\
\hline 24 & 4 & $7.3 \times 10^{4}$ & 5 & $7.6 \times 10^{4}$ & 5 & WMA & 17 & WMA & $\mathrm{P}$ & $\mathrm{P}$ & \\
\hline 25 & 4 & $9.2 \times 10^{4}$ & 6 & $1.1 \times 10^{6}$ & 4 & WMA & 17 & WMA & $\mathrm{P}$ & $\mathrm{P}$ & \\
\hline 26 & 4 & $7.9 \times 10^{5}$ & 8 & $1.5 \times 10^{7}$ & 9 & WMA & 18 & WMA & $\mathrm{P}$ & $\mathrm{P}$ & \\
\hline 27 & 2 & $3.5 \times 10^{4}$ & 6 & $3.5 \times 10^{5}$ & 10 & WMA & 18 & Normal & $P$ & $P$ & \\
\hline 28 & 4 & $3.6 \times 10^{5}$ & 8 & $3.4 \times 10^{6}$ & 11 & WMA & 17 & Normal & $P$ & $P$ & \\
\hline 29 & 1 & $4.0 \times 10^{7}$ & 9 & $1.8 \times 10^{6}$ & 13 & WMA & 17 & Normal & $\mathrm{P}$ & $\mathrm{P}$ & \\
\hline 30 & 4 & $9.6 \times 10^{4}$ & 5 & $1.1 \times 10^{7}$ & 8 & WMA & 19 & Normal & $\mathrm{P}$ & $\mathrm{P}$ & \\
\hline
\end{tabular}


Table 1 Continued

\begin{tabular}{|c|c|c|c|c|c|c|c|c|c|c|c|}
\hline \multirow[b]{2}{*}{$\begin{array}{l}\text { Pt } \\
\text { no }\end{array}$} & \multicolumn{2}{|c|}{$\begin{array}{l}\text { Screening for } \\
\text { CMV }\end{array}$} & \multicolumn{2}{|c|}{$\begin{array}{l}\text { Re-examination of } \\
\text { CMV }\end{array}$} & \multicolumn{2}{|c|}{ First MRI examination } & \multicolumn{2}{|c|}{ Second MRI examination } & \multicolumn{2}{|l|}{ AABR } & \multirow[b]{2}{*}{ Outcome* } \\
\hline & $\begin{array}{l}\text { Age } \\
\text { (days) }\end{array}$ & $\begin{array}{l}\text { Copies/ } \\
\mathrm{mL}\end{array}$ & $\begin{array}{l}\text { Age } \\
\text { (weeks) }\end{array}$ & $\begin{array}{l}\text { Copies/ } \\
\mathrm{mL}\end{array}$ & $\begin{array}{l}\text { Age } \\
\text { (weeks) }\end{array}$ & Findings & $\begin{array}{l}\text { Age } \\
\text { (months) }\end{array}$ & Findings & Right & Left & \\
\hline 31 & 1 & $1.1 \times 10^{6}$ & 9 & $2.1 \times 10^{5}$ & 11 & WMA & 18 & Normal & ND & ND & \\
\hline 32 & 1 & $1.6 \times 10^{8}$ & 4 & $6.9 \times 10^{7}$ & 5 & WMA & Declined & & $\mathrm{P}$ & $\mathrm{P}$ & \\
\hline 33 & 4 & $1.9 \times 10^{5}$ & 9 & $6.6 \times 10^{5}$ & 14 & WMA & Declined & & $P$ & $\mathrm{P}$ & \\
\hline 34 & 4 & $5.1 \times 10^{6}$ & 15 & $2.9 \times 10^{5}$ & 7 & WMA & Not yet do & & $P$ & $\mathrm{P}$ & \\
\hline 35 & 4 & $3.7 \times 10^{5}$ & 8 & $3.4 \times 10^{5}$ & 8 & WMA & Declined & & $\mathrm{P}$ & $\mathrm{P}$ & \\
\hline 36 & 4 & $1.1 \times 10^{4}$ & 6 & $4.8 \times 10^{5}$ & 8 & WMA & Not yet do & & $P$ & $P$ & \\
\hline 37 & 1 & $3.9 \times 10^{4}$ & 6 & $1.3 \times 10^{5}$ & 5 & WMA & Not yet do & & ND & ND & \\
\hline 38 & 1 & $1.5 \times 10^{7}$ & \multicolumn{2}{|c|}{ No urine collection } & 7 & WMA & Not yet do & & $P$ & $\mathrm{P}$ & \\
\hline 39 & 4 & $6.9 \times 10^{7}$ & 15 & $9.8 \times 10^{6}$ & 14 & Normal & 20 & WMA & $\mathrm{P}$ & $P$ & \\
\hline 40 & 1 & $1.1 \times 10^{9}$ & 16 & $2.0 \times 10^{7}$ & 18 & Normal & 18 & WMA & $\mathrm{P}$ & $P$ & \\
\hline 41 & 1 & $3.5 \times 10^{7}$ & 10 & $6.2 \times 10^{6}$ & 13 & Normal & 17 & WMA & $P$ & $P$ & \\
\hline 42 & 4 & $6.7 \times 10^{5}$ & 8 & $6.2 \times 10^{5}$ & 10 & Normal & 24 & WMA & $\mathrm{P}$ & $P$ & \\
\hline 43 & 4 & $3.6 \times 10^{5}$ & 9 & $4.7 \times 10^{6}$ & 11 & Normal & 22 & WMA & $P$ & $P$ & Cavum vergae \\
\hline 44 & 2 & $1.4 \times 10^{7}$ & 8 & $4.4 \times 10^{5}$ & 9 & Normal & 18 & WMA & ND & ND & \\
\hline 45 & 4 & $6.1 \times 10^{4}$ & 8 & $6.1 \times 10^{4}$ & 7 & Normal & 16 & Normal & $P$ & $P$ & \\
\hline 46 & 4 & $3.8 \times 10^{4}$ & 11 & $2.2 \times 10^{6}$ & 16 & Normal & 18 & Normal & $P$ & $P$ & \\
\hline 47 & 4 & $1.2 \times 10^{6}$ & 12 & $1.6 \times 10^{7}$ & 12 & Normal & 17 & Normal & $P$ & $P$ & \\
\hline 48 & 1 & $4.5 \times 10^{3}$ & 4 & $3.1 \times 10^{5}$ & 4 & Normal & Declined & & $P$ & $P$ & \\
\hline 49 & 1 & $6.4 \times 10^{6}$ & 14 & $4.1 \times 10^{5}$ & 15 & Normal & Declined & & $\mathrm{P}$ & $P$ & \\
\hline 50 & 4 & $7.4 \times 10^{5}$ & 13 & $2.2 \times 10^{6}$ & 28 & Normal & Declined & & $P$ & $P$ & \\
\hline 51 & 4 & $6.1 \times 10^{4}$ & 3 & $1.2 \times 10^{5}$ & 5 & Normal & Declined & & $\mathrm{P}$ & $P$ & \\
\hline 52 & 4 & $1.1 \times 10^{6}$ & 13 & $6.0 \times 10^{6}$ & 12 & Normal & Not yet do & & $P$ & $\mathrm{P}$ & \\
\hline 53 & 4 & $9.3 \times 10^{5}$ & 7 & $3.9 \times 10^{6}$ & 8 & Normal & Not yet do & & $\mathrm{P}$ & $\mathrm{P}$ & \\
\hline 54 & 1 & $1.1 \times 10^{4}$ & \multicolumn{2}{|c|}{ Not examined ${ }^{\star *}$} & \multicolumn{2}{|c|}{ Not examined } & \multicolumn{2}{|c|}{ Not examined } & $\mathrm{P}$ & $\mathrm{P}$ & \\
\hline 55 & 1 & $3.1 \times 10^{6}$ & \multicolumn{2}{|c|}{ Not examined } & \multicolumn{2}{|c|}{ Not examined } & \multicolumn{2}{|c|}{ Not examined } & $P$ & $\mathrm{P}$ & \\
\hline 56 & 3 & $2.4 \times 10^{6}$ & \multicolumn{2}{|c|}{ Not examined } & \multicolumn{2}{|c|}{ Not examined } & \multicolumn{2}{|c|}{ Not examined } & $\mathrm{P}$ & $\mathrm{P}$ & \\
\hline 57 & 4 & $7.7 \times 10^{6}$ & \multicolumn{2}{|c|}{ Not examined } & \multicolumn{2}{|c|}{ Not examined } & \multicolumn{2}{|c|}{ Not examined } & $P$ & $P$ & \\
\hline 58 & 4 & $2.4 \times 10^{3}$ & \multicolumn{2}{|c|}{ Not examined } & \multicolumn{2}{|c|}{ Not examined } & \multicolumn{2}{|c|}{ Not examined } & $\mathrm{P}$ & $\mathrm{P}$ & \\
\hline 59 & 1 & $1.0 \times 10^{4}$ & \multicolumn{2}{|c|}{ Not examined } & \multicolumn{2}{|c|}{ Not examined } & \multicolumn{2}{|c|}{ Not examined } & ND & ND & \\
\hline 60 & 2 & $1.1 \times 10^{8}$ & \multicolumn{2}{|c|}{ Not examined } & Not exam & ined & Not exami & & ND & ND & \\
\hline
\end{tabular}

Microcephaly was noted in one newborn with modified HC z score of -2.95 .

*No data means the patient had shown no apparent clinical manifestations until documentation of this paper.

†The oral antiviral drug valganciclovir was administered to patients 1-5 with SNHL for 5-6 months. Patient 5 presented with late-onset hearing loss and was then treated with valganciclovir. †Figures in parentheses indicate hearing loss measured in decibel (dB).

§Calcification was confirmed with CT scan.

INot yet done means that the second MRI examination is scheduled but had not yet been done until documentation of this paper.

${ }^{* *}$ Not examined means no visit or examinations.

AABR, automatic auditory brainstem response; HC, nead circumference; nt, not tested; P, pass; PDD, psychomotor developmental delays; Pt no, patient number; R, refer; SNHL, sensorineural hearing loss; WMA, white matter abnormality. 

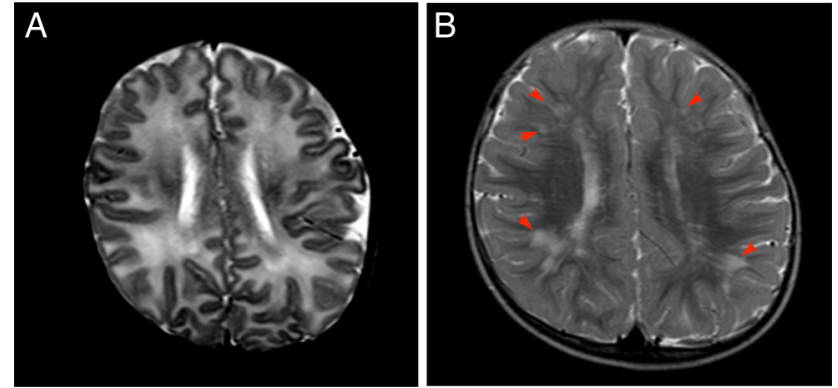

Figure $1 \mathrm{MRI}$ findings in patient number 4 with congenital cytomegalovirus infection at the age of 1 month $(A)$ and at the age of 18 months (B). (A) Axial T2-weighted images show diffuse abnormal high intensity. (B) Follow-up axial

T2-weighted images at the age of 18 months show residual abnormal T2 high intensity areas (arrowheads).

$\mathrm{p}=0.036$, Mann-Whitney $\mathrm{U}$ test). When newborns were retested at a mean age of 7.7 weeks (range 2-16 weeks), the urinary CMV DNA copy number of newborns with SNHL $\quad\left(9.90 \times 10^{7}\right.$ copies $/ \mathrm{mL}, 95 \%$ CI $2.44 \times 10^{7}$ to $4.02 \times 10^{8}$ copies $/ \mathrm{mL}$ ) was still significantly higher than in newborns who had cCMV infection but no SNHL $\left(2.61 \times 10^{6}\right.$ copies $/ \mathrm{mL}, 95 \%$ CI $2.88 \times 10^{4}$ to $2.37 \times 10^{8}$ copies $/ \mathrm{mL} ; \mathrm{p}=0.010$ ) (figure 4 ).

\section{Relationship between cCMV infection and MRI findings}

Of the 60 newborns with cCMV, 53 underwent MRI scans. Of these 53 newborns, $44(83.0 \%)$ were found to have some form of CNS damage (figure 5). Fifty of the 53 newborns underwent an initial MRI scan before the age of 5 months; two of the remaining three newborns underwent the scan at 7 or 10 months while the other was scanned at 18 months. Of the 50 newborns who underwent MRI scans by the age of 6 months, 36 were found to have some form of CNS damage $(72.0 \%)$. The mean urinary CMV DNA copy number of newborns who were found to have CNS damage during screening $\left(2.30 \times 10^{6}\right.$ copies $/ \mathrm{mL}, 95 \%$ CI $8.78 \times 10^{5}$ to $6.01 \times 10^{6}$ copies $/ \mathrm{mL}$ ) did not differ significantly from that in 14 newborns who had no such abnormalities $\left(1.31 \times 10^{6}\right.$ copies $/ \mathrm{mL}, 95 \%$ CI $1.90 \times 10^{5}$ to $9.07 \times 10^{6}$ copies $\left./ \mathrm{mL}\right)$ (figure 6A).

Thirty-one newborns underwent a second scan at the age of 18 months (range 16-20 months). During the second MRI scan, 23 newborns were found to have CNS damage $(74.2 \%)$ and these newborns had higher urinary CMV DNA copy numbers $\left(6.19 \times 10^{6}\right.$ copies $/ \mathrm{mL}$, $95 \%$ CI $1.90 \times 10^{6}$ to $2.02 \times 10^{7}$ copies $/ \mathrm{mL}$ ) than the eight newborns in which no such abnormalities were detected $\left(3.32 \times 10^{5}\right.$ copies $/ \mathrm{mL}, 95 \%$ CI $4.48 \times 10^{4}$ to $2.46 \times 10^{6}$ copies $/ \mathrm{mL} ; \mathrm{p}=0.013$ ) (figure $6 \mathrm{~B}$ ).

The MRI scans performed before 6 months of age and around the age of 18 months were compared for 30 infants. The mean CMV DNA copy number of the 18 newborns who were found to have abnormal CNS findings in the first and second scans $\left(3.48 \times 10^{6}\right.$ copies $/ \mathrm{mL}$,
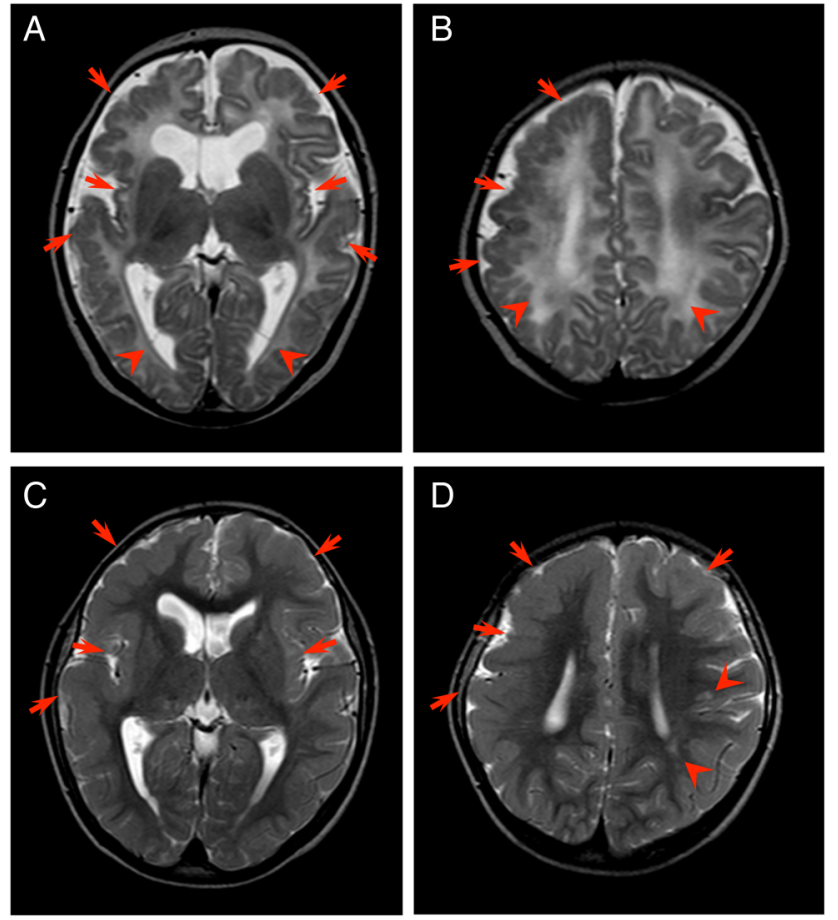

Figure $2 \mathrm{MRI}$ findings in patient number 6 with congenital cytomegalovirus infection at the age of 3 months (A and $B$ ) and at the age of 18 months (C and D). (A) Axial T2-weighted images at the level of the basal ganglia show polymicrogyria involving bilateral frontal lobes and perisylvian and insular cortices (arrows) and bilateral occipital ventricular septations (arrowheads). Bilateral enlargement of the lateral ventricles shows volume loss of white matter. (B) Axial T2-weighted images at the level of the central semiovale show right predominant frontal lobe polymicrogyria (arrows) and diffuse abnormal T2 high intensity areas (arrowheads). (C, D) Follow-up MRI study at the age of 18 months also shows bilateral polymicrogyria (arrows), but abnormal T2 high intensity has been obscured and there are only some scattered abnormal T2 high intensity areas (arrowheads). Abnormally thin centrum semiovale suggests white matter volume loss.

$95 \%$ CI $9.14 \times 10^{5}$ to $1.32 \times 10^{7}$ copies $/ \mathrm{mL}$ ) was significantly higher than in three newborns with normal CNS findings in both scans $\left(1.41 \times 10^{5}\right.$ copies $/ \mathrm{mL}, 95 \%$ CI $1.35 \times 10^{3}$ to $1.47 \times 10^{7}$ copies $\left./ \mathrm{mL} ; \mathrm{p}=0.035\right)$. Five newborns were found to have WMAs on an initial scan but no abnormalities were detected on the second scan. The urinary CMV DNA copy number of these five newborns $\left(5.56 \times 10^{5}\right.$ copies $/ \mathrm{mL}, 95 \%$ CI $1.89 \times 10^{4}$ to $1.64 \times 10^{7}$ copies $/ \mathrm{mL}$ ) did not differ significantly from that of newborns without CNS abnormalities in both scans $(\mathrm{p}=0.67)$. In contrast, four newborns had no abnormalities initially but were found to have WMAs on the second scan. In these four newborns, the urinary CMV DNA copy number was significantly higher $\left(7.81 \times 10^{7}\right.$ copies $/ \mathrm{mL}, 95 \%$ CI $3.92 \times 10^{6}$ to $1.56 \times 10^{9}$ copies $/ \mathrm{mL}$ ) than the three infants who had no abnormalities $(p=0.034)$ (figure 7). All five newborns with SNHL $(100 \%)$ and 39 of 48 children without SNHL $(81 \%)$ had 


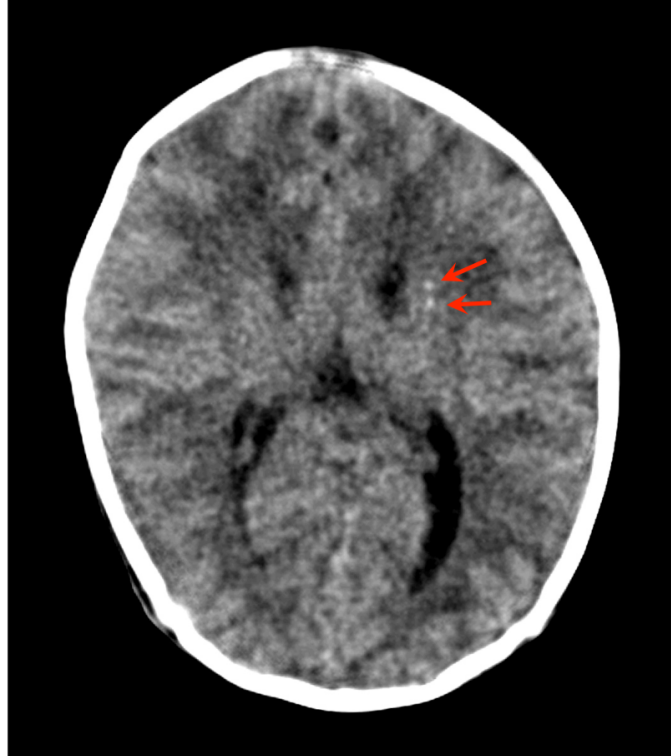

Figure 3 Basal ganglia calcification. Unenhanced CT image of patient number 2 shows punctate calcification in the head of the left caudate nucleus (arrows).
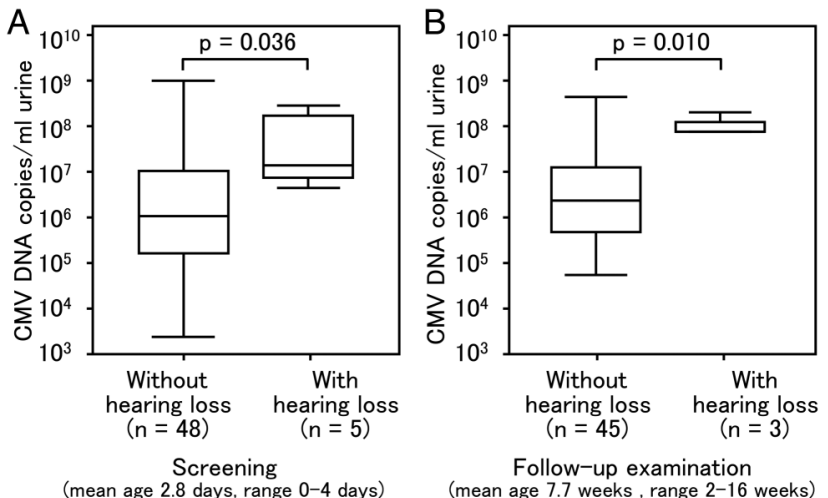

Figure 4 Comparison of cytomegalovirus (CMV) load in urine samples of newborns with congenital CMV (cCMV) infection and hearing loss and in those of newborns with cCMV infection but no hearing loss. DNA was extracted from urine samples collected from newborns within 0-5 days of birth and CMV DNA was quantified by quantitative real-time PCR. (A, B) Number of copies of CMV in 48 newborns with CMV infection but no hearing loss (without hearing loss) and five newborns with CMV infection and hearing loss (with hearing loss) in the screening $(p=0.036)$ and in the follow-up examination $(p=0.010)$. Upper and lower borders of box plots represent the 25th and 75th percentiles and bars in the boxes represent median viral loads. Upper and lower bars indicate maximum and minimum viral load, respectively.

abnormal CNS findings including WMAs, thus the incidences of abnormal CNS findings were not significantly different between the two groups $(p=0.288)$. Children with SNHL, however, showed significantly far more abnormal CNS findings other than WMAs (3/5 with SNHL) than children without SNHL (5/48 without SNHL) $(\mathrm{p}=0.003)$ (table 1$)$.

\section{DISCUSSION}

This study examined the incidence of cCMV infection and SNHL in seemingly healthy newborns with no obvious clinical manifestations of cCMV infection. One of 60 newborns $(1.7 \%)$ who tested positive for CMV exhibited microcephaly but no other clinical manifestations. As microcephaly has been reported to be a common single clinical finding in newborns with cCMV infection, ${ }^{17}$ nearly asymptomatic newborns with milder or non-specific findings can be missed in the absence of further screening. Therefore, this study used MRI to identify CNS damage in newborns with cCMV infection and examined the relationship between CNS damage and urinary CMV DNA copy number.

Various methods have been used to screen for cCMV infection. ${ }^{18-20}$ However, no previous study for cCMV screening has described the direct extraction of CMV DNA from the liquid urine of newborns. We subjected CMV DNA to qPCR and screened for cCMV infection in over 23000 newborns. Unlike blood, urine can be collected non-invasively and the viral load in urine is likely to be elevated even when a virus is inactive. Real-time PCR was used to detect CMV DNA in urine samples in order to ensure specificity, quantitative reliability and rapidity. The method used in this study also allows accurate quantitative determination of urinary volume, unlike the technique employing FTA-Elute filter cards described in previous studies. ${ }^{8}$ The real-time PCR assay of saliva has excellent sensitivity and specificity and can easily be adapted for large-scale screening of newborns. ${ }^{19}$ However, the real-time PCR assay of saliva is less able to provide the quantitative capability than that of liquid urine. Thus, the novel method described here allows more accurate examination of the relationship between viral load and SNHL/CNS damage.

In this study the incidence of cCMV infection was $0.257 \%$, about $17 \%$ lower than that reported in other studies conducted in Japan. Numazaki $e t a l^{7}$ used a conventional cell culture method to screen 11938 individuals and reported an incidence of $0.31 \%$, whereas Koyano $e t a l^{8}$ used FTA-Elute filter cards embedded in a diaper to screen 21272 individuals and reported an incidence of $0.31 \%$. The higher level of cCMV infection detected in these studies could be explained by the fact that they recruited newborns from university hospitals, a population likely to include newborns at a greater risk of cCMV infection. In fact, Koyano $e t a l^{8}$ reported that the incidence of cCMV infection at primary obstetric clinics was $0.24 \%$. Our sample of seemingly healthy newborns, born at typical maternity hospitals, might provide a CMV incidence that is closer to the typical incidence of cCMV infection.

Newborn hearing screening was performed using AABR testing. Of the screened newborns, 171 were referred for further testing and $22(12.9 \%)$ were finally diagnosed with SNHL. Four newborns underwent both newborn hearing screening and screening for cCMV infection, and all four of these newborns and one other 


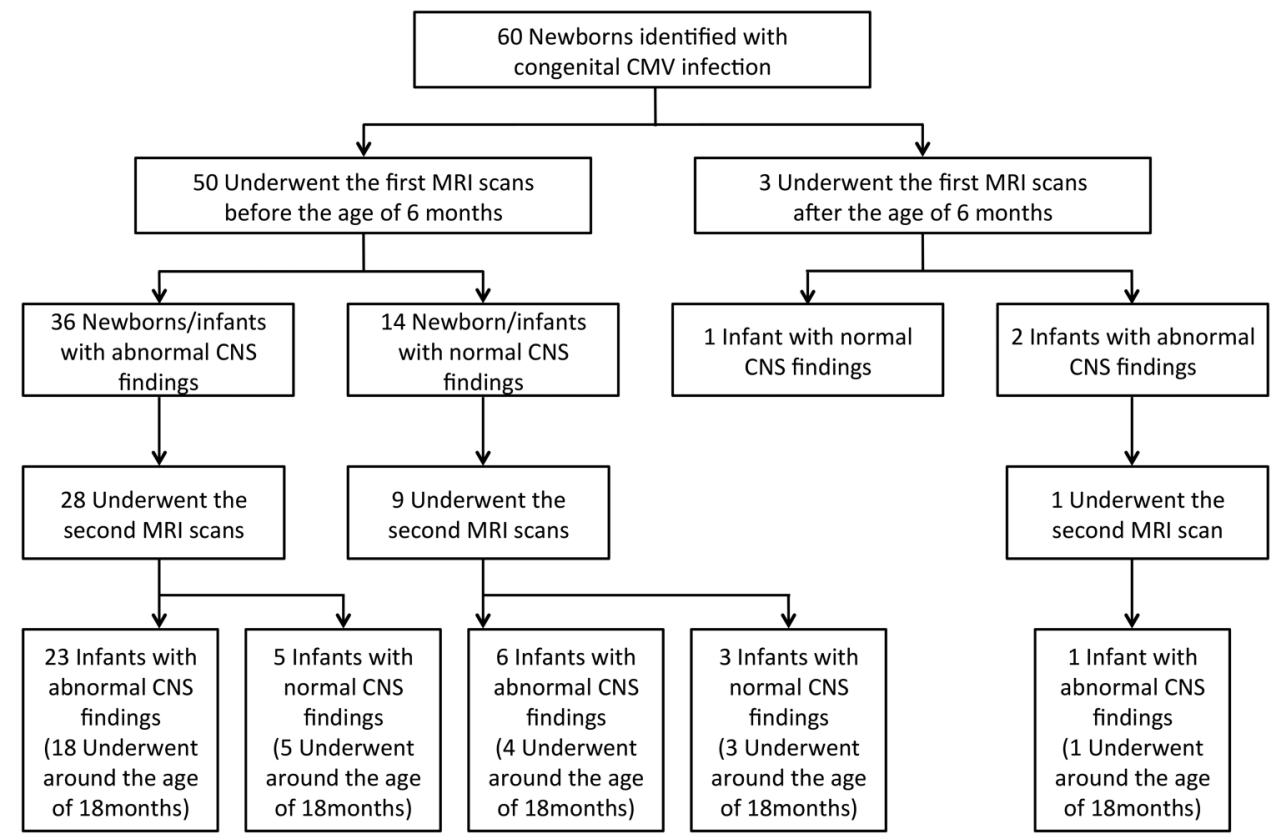

Figure 5 Enrolment in the head MRI scan and outcomes. 'Around the age of 18 months' means that the second MRI scans were performed between the ages of 16 months and 20 months.

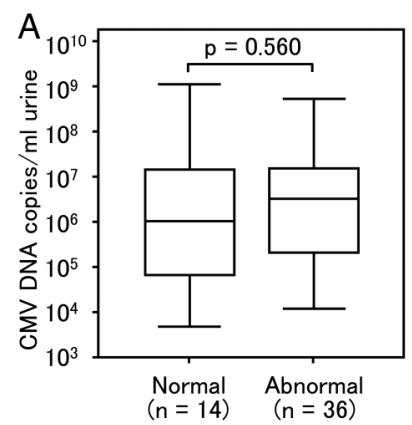

The first examination

(mean age 2.2 months, range $0.7-4.2$ months) (mean age 18 months, range $16-20$ months)

Figure 6 Comparison of urinary cytomegalovirus (CMV) screening based on the difference in MRI manifestations in congenital CMV infection. Urine samples were collected from newborns within 0-5 days of birth. (A) Of the 60 newborns, 53 underwent MRI examination. Three newborns had the first MRI scans carried out much later than the other 50 at 7, 10 and 18 months, respectively 50 . Thus, three were excluded from the comparison. Of the 50 newborns on whom MRI was performed at less than 5 months of age, 14 (normal) did not have central nervous system (CNS) damage and 36 (abnormal) had CNS damage. No significant difference in the viral load is shown between normal and abnormal $(p=0.560)$.

(B) Thirty-one newborns underwent a second MRI scan between 16 months and 20 months of age. Twenty-three newborns (abnormal) were found to have CNS damage during the second scan and eight newborns (normal) had no such abnormalities. A significant difference in the viral load is shown between normal and abnormal $(p=0.013)$. Upper and lower borders of box plots represent the 25th and 75th percentiles and bars represent median viral loads. Upper and lower bars indicate maximum and minimum viral load, respectively. newborn with late-onset $\mathrm{HL}$ had SNHL. In this newborn, because the AABR test was not conducted within 5 days of birth, ABR was performed but HL was not confirmed. HL detected during follow-up was diagnosed as late-onset HL. Thus, performing hearing screening and CMV screening at the same time should allow early prediction of SNHL prior to more detailed hearing testing. In other words, simultaneous screening may allow early detection and treatment of SNHL due to cCMV infection.

In 60 newborns with cCMV infection, those with the highest urinary CMV DNA copy number had $4.6 \times 10^{5}$-fold more copies than those with the lowest $\mathrm{CMV}$ DNA copy number (range $2.4 \times 10^{3}-1.1 \times 10^{9}$ copies $/ \mathrm{mL}$ ). Newborns with cCMV infection and SNHL had a significantly higher urinary CMV DNA copy number than those with cCMV infection but no SNHL (figure 4). Of the 60 newborns with cCMV infection, one had late-onset SNHL. It is well known that SNHL due to cCMV infection appears as a form of late auditory dysfunction. $^{2122}$ Thus, infants with cCMV infection, particularly with high urinary CMV DNA copy number, need to be carefully monitored for the development of late-onset SNHL.

Of the 60 newborns with cCMV infection, 53 underwent MRI. Abnormal findings were noted in 44 of these infants $(83.0 \%)$, and most of the findings were WMAs that have been attributed to myelination failure induced by CMV infection of the CNS. ${ }^{5} 1423$

The first MRI examination performed by the age of 6 months identified no significant difference between the urinary CMV DNA copy number of newborns with normal MRI findings and those with abnormal MRI 
findings. The second examination carried out at about the age of 18 months showed that newborns with abnormal MRI findings had a significantly higher urinary CMV DNA copy number than those with normal MRI findings (figure 6). High CMV DNAemia has been reported to predict CMV infection sequelae in newborns with asymptomatic cCMV infection, ${ }^{24}$ so high urinary CMV DNA copy numbers could predict late CNS damage.

The urinary CMV viral load of newborns with initial abnormal results who subsequently had normal results did not differ significantly from that of newborns without abnormal results. Conversely, the urinary CMV viral load of newborns who initially had normal results but subsequently had abnormal results was significantly higher than that of newborns who had normal results (figure 7). Newborns with a high urinary CMV DNA copy number during screening should be followed up with MRI.

In this study, MRI examination revealed that WMAs occurred with surprising frequency of $83 \%$ in newborns and infants with cCMV infection. Although nearly half of WMAs found in the children with cCMV infection were considered to be mild, WMAs could be the critical sign of asymptomatic cCMV infection detectable with neuroimaging examination. WMAs are very often seen in children with symptomatic cCMV infection. ${ }^{6} 232526$ Uematsu et $a l^{27}$ retrospectively investigated asymptomatic cCMV infection with neurological sequelae using qPCR of CMV DNA from dried umbilical cord and reported that all the 54 patients $(100 \%)$ had cerebral WMAs in MRI study. In contrast, there are very few references about the incidence of WMAs in newborns and infants with cCMV infection found through neonatal screening.

Recently, Krakar et $a t^{28}$ described the apparent regression of WMAs in a case report of serial postnatal MRIs in a child and proposed that leukoencephalopathy in cCMV infection is not only non-progressive or static as previously reported, but also might even evolve in part of WMAs, suggesting both disturbed and delayed myelination. Consequently, it could be considered that most WMAs due to cCMV infection, especially mild WMAs, would regress or disappear in late childhood, although many WMAs of varying severity and extent exist in the neonatal period and infancy. However, the qualitative and quantitative evaluations of WMAs caused by cCMV infection remain to be well-defined.

Milewska-Bobula et $a l^{29}$ indicated increased emotional sensitivity and problems with school maturity in 6-year-old children with asymptomatic or mildly symptomatic cCMV infection, and Karltorp et $a l^{30}$ showed that $88 \%$ of 26 children with congenital CMV infection had balance disturbances including walking at a later age. Whether WMAs in cCMV infection cause a subsequent developmental disorder is still unclear, ${ }^{14} 25{ }^{26}$ and newborns and infants with SNHL in our study showed far more abnormal CNS findings other than WMAs than those without SNHL; thus SNHL could occur in children with more severely damaged CNS. WMAs in

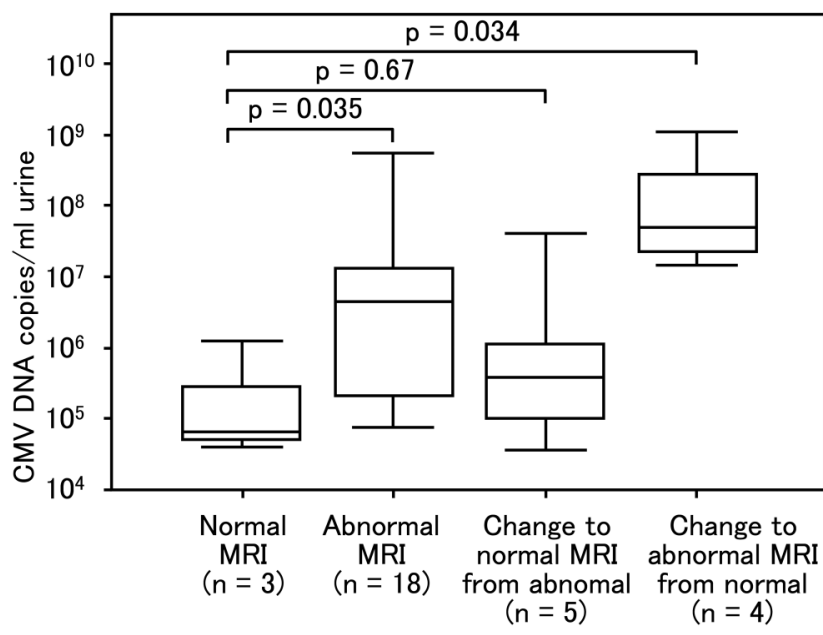

Figure 7 Comparison of cytomegalovirus (CMV) load in urine samples of newborns with congenital CMV infection with respect to the changes in MRI findings. Urine samples were collected from newborns within 0-5 days of birth. The first $\mathrm{MRI}$ scans were performed at less than 5 months of age. The second MRI scans were performed between the age of 16 months and 20 months. One of 31 newborns who underwent the first and second MRI scans was excluded from the comparison because the first MRI scan was performed at 10 months of age. In the first and second MRI scans, three newborns (normal) showed no abnormalities while 18 newborns (abnormal) showed central nervous system damage. The initial scan revealed abnormalities but the second scan showed no abnormalities in five newborns (changed to normal). In contrast, the initial scan revealed no abnormalities but the second scan showed abnormalities in four newborns (change to abnormal). The 18 (abnormal MRI) and four newborns (change to abnormal MRI from normal) who had abnormalities in the second MRI scans showed a significant difference $(p=0.035$ and $p=0.034$, respectively) compared with three newborns (normal) who had no abnormalities in both the first and second MRI scans while the five newborns who had no abnormalities in the second MRI scans showed no significant difference $(p=0.67)$ compared with newborns with no abnormalities in both the first and second MRI scans. Upper and lower borders of box plots represent the 25th and 75th percentiles and bars represent median viral loads. Upper and lower bars indicate maximum and minimum viral load, respectively.

newborns and infants could be related to the subsequent developmental disorders such as balance disturbances and emotional problems, aside from the well-known clinical manifestations such as intellectual disability and cerebral palsy that could be referred to the severe CNS damage.

Further follow-up of the patients in this study is therefore needed, and the relationship between the site where a WMA was noted, the severity of WMA, the CMV DNA copy number in the urine and the occurrence of developmental disorders needs to be examined in greater detail.

This study reports the incidence of cCMV infection and the typical range of urinary CMV viral load of 
seemingly healthy newborns in Japan. Newborns with cCMV infection and SNHL had a higher urinary CMV viral load. Newborns who had CNS abnormalities including WMAs detected by MRI also had a significantly higher urinary CMV viral load. Because the urinary CMV viral load could be directly related to illness, the use of qPCR to determine the CMV viral load in urine specimens may identify cCMV infection and predict its sequelae. However, this study has an important limitation in the implementation. Urine collection from newborns is laborious and time-consuming compared with a saliva swab or a FTA-Elute filter card embedded in a diaper, and thus the use of qPCR for a urine sample as a screening test requires improvements in usability and economic efficiency. The saliva swab or FTA-Elute filter card would be better suited for the screening of cCMV infection. The CMV viral load in liquid urine from a newborn found to be positive by screening should then be determined to assess CNS abnormalities, predict sequelae or follow late-onset HL.

\author{
Author affiliations \\ ${ }^{1}$ Laboratory of Clinical Research, Saitama Children's Medical Center, Saitama, \\ Japan \\ 2Department of Radiological Technology, Saitama Children's Medical Center, \\ Saitama, Japan \\ ${ }^{3}$ Division of Infectious Disease, Saitama Children's Medical Center, Saitama, \\ Japan \\ ${ }^{4}$ Division of Otorhinolaryngology, Kawagoe Otology Institute, Saitama, Japan \\ ${ }^{5}$ Mejiro University Audiology Clinic, Saitama, Japan \\ ${ }^{6}$ Division of Otolaryngology, Saitama Children's Medical Center, Saitama, \\ Japan \\ ${ }^{7}$ Division of Radiology, Saitama Children's Medical Center, Saitama, Japan \\ ${ }^{8}$ Division of Pediatrics, Sannoh Doom Clinic, Saitama, Japan \\ ${ }^{9}$ Division of Obstetrics and Gynecology, Sannoh Clinic, Saitama, Japan \\ ${ }^{10}$ Division of Obstetrics and Gynecology, Aiwa Hospital, Saitama, Japan \\ ${ }^{11}$ Department of Molecular Predictive Medicine and Sport Science, School of \\ Medicine, Kyorin University, Tokyo, Japan
}

Acknowledgements The authors would like to thank the newborns and their parents who agreed to take part in the study and medical colleagues who collaborated in this study.

Contributors TO and HS designed the study. AY and TO participated in data analysis and writing the manuscript. AY and TA conducted all initial urine screening. HS, NA and SA diagnosed hearing loss. EO performed MRI and CT image analysis. HK, JM, HF, TU, JF and KS enrolled newborns and contributed to hearing screening. KS, HO and TK contributed to critical revision of the manuscript.

Funding This study was supported by grants for the Research on Child Development and Diseases (H20-Kodomo-007; H23-Jisedai-Ippan-001) from the Ministry of Health, Labour and Welfare, Japan.

Competing interests None declared.

Patient consent Patient consents were obtained from the parents.

Ethics approval Ethics approval was provided by the ethical committees of the participating hospitals.

Provenance and peer review Not commissioned; externally peer reviewed.

Data sharing statement No additional data are available.

Open Access This is an Open Access article distributed in accordance with the Creative Commons Attribution Non Commercial (CC BY-NC 4.0) license, which permits others to distribute, remix, adapt, build upon this work noncommercially, and license their derivative works on different terms, provided the original work is properly cited and the use is non-commercial. See: http:// creativecommons.org/licenses/by-nc/4.0/

\section{REFERENCES}

1. Stagno S, Whitley RJ. Herpesvirus infection of pregnancy. Part I: Cytomegalovirus and Epstein-Barr virus infections. N Engl J Med 1985;313:1270-4.

2. Morton CC, Nance WE. Newborn hearing screening--a silent revolution. N Engl J Med 2006;354:2151-64.

3. Fowler K, McCollister F, Dahle A, et al. Progressive and fluctuating sensorineural hearing loss in children with asymptomatic congenital cytomegalovirus infection. J Pediatr 1997;130:624-30.

4. Hicks T, Fowler K, Richardson M, et al. Congenital cytomegalovirus infection and neonatal auditory screening. $J$ Pediatr 1993;123:779-82.

5. van der Knaap MS, Vermeulen G, Barkhof $F$, et al. Pattern of white matter abnormalities at MR imaging: use of polymerase chain reaction testing of Guthrie cards to link pattern with congenital cytomegalovirus infection. Radiology 2004;230:529-36.

6. de Vries LS, Gunardi H, Barth PG, et al. The spectrum of cranial ultrasound and magnetic resonance imaging abnormalities in congenital cytomegalovirus infection. Neuropediatrics 2004:35:113-19.

7. Numazaki K, Fujikawa T. Chronological changes of incidence and prognosis of children with asymptomatic congenital cytomegalovirus infection in Sapporo, Japan. BMC Infect Dis 2004:4:22.

8. Koyano S, Inoue N, Oka A, et al., Japanese Congenital Cytomegalovirus Study Group. Screening for congenital cytomegalovirus infection using newborn urine samples collected on filter paper: feasibility and outcomes from a multicentre study. BMJ Open 2011;1:e000118.

9. Noyola DE, Demmler GJ, Nelson CT, et al. Early predictors of neurodevelopmental outcome in symptomatic congenital cytomegalovirus infection. J Pediatr 2001;138:325-31.

10. Nance WE, Lim BG, Dodson KM. Importance of congenital cytomegalovirus infections as a cause for pre-lingual hearing loss. $J$ Clin Virol 2006;35:221-5.

11. Wirgart BZ, Andersson P, Grillner L. Evaluation of the ReSSQ assay in relation to the COBAS AMPLICOR CMV MONITOR test and an in-house nested PCR method for detection of cytomegalovirus DNA. J Clin Microbiol 2005;43:4057-63.

12. Griscelli F, Barrois M, Chauvin S, et al. Quantification of human cytomegalovirus DNA in bone marrow transplant recipients by real-time PCR. J Clin Microbiol 2001;39:4362-9.

13. Kelley PM, Harris DJ, Comer BC, et al. Novel mutations in the connexin 26 gene (GJB2) that cause autosomal recessive (DFNB1) hearing loss. Am J Hum Genet 1998;62:792-9.

14. Fink KR, Thapa MM, Ishak GE, et al. Neuroimaging of pediatric central nervous system cytomegalovirus infection. Radiographics 2010;30:1779-96.

15. Pascual-Castroviejo I, Pascual-Pascual SI, Velasquez-Fragua R, et al. Congenital cytomegalovirus infection and cortical/subcortical malformations. Neurologia 2012;27:336-42.

16. van der Voorn JP, Pouwels PJ, Vermeulen RJ, et al Quantitative MR imaging and spectroscopy in congenital cytomegalovirus infection and periventricular leukomalacia suggests a comparable neuropathological substrate of the cerebral white matter lesions. Neuropediatrics 2009;40:168-73.

17. Dreher AM, Arora N, Fowler KB, et al. Spectrum of disease and outcome in children with symptomatic congenital cytomegalovirus infection. J Pediatr 2014;164:855-9.

18. Kenneson A, Cannon MJ. Review and meta-analysis of the epidemiology of congenital cytomegalovirus (CMV) infection. Rev Med Virol 2007:17:253-76.

19. Boppana SB, Ross SA, Shimamura M, et al., Saliva polymerasechain-reaction assay for cytomegalovirus screening in newborns. N Engl J Med 2011;364:2111-18.

20. Paixão P, Almeida S, Videira PA, et al. Screening of congenital cytomegalovirus infection by real-time PCR in urine pools. Eur $J$ Pediatr 2012:171:125-9.

21. Foulon I, Naessens A, Foulon W, et al. A 10-year prospective study of sensorineural hearing loss in children with congenital cytomegalovirus infection. J Pediatr 2008;153:84-8.

22. Amir J, Attias J, Pardo J. Treatment of late-onset hearing loss in infants with congenital cytomegalovirus infection. Clin Pediatr (Phila) 2014:53:444-8

23. Barkovich AJ, Lindan CE. Congenital cytomegalovirus infection of the brain: imaging analysis and embryological considerations. AJNR Am J Neuroradiol 1994;15:703-15. 
24. Forner G, Abate D, Mengoli C, et al. High cytomegalovirus (CMV) DNAemia predicts CMV sequelae in asymptomatic congenitally infected newborns born to women with primary infection during pregnancy. J Infect Dis 2015;212:67-71.

25. Manara R, Balao L, Baracchini C, et al. Brain magnetic resonance findings in symptomatic congenital cytomegalovirus infection. Pediatr Radiol 2011;41:962-70.

26. Inder TE, Wells SJ, Mogridge NB, et al. Defining the nature of the cerebral abnormalities in the premature infant: a qualitative magnetic resonance imaging study. J Pediatr 2003;143:171-9.

27. Uematsu M, Haginoya K, Kikuchi A, et al. Asymptomatic congenital cytomegalovirus infection with neurological sequelae: a retrospective study using umbilical cord. Brain Dev 2016;38:819-26.

28. Krakar G, Dakovic I, Delin S, et al. Evolutive leukoencephalopathy in congenital cytomegalovirus infection. J Child Neurol

2015;30:93-5.

29. Milewska-Bobula B, Zebrowska J, Olszaniecka M, et al. Evaluation of intellectual development of children following congenital, mildly symptomatic cytomegalovirus (CMV) infection. A prospective study. Med Wieku Rozwoj 2010;14:370-3.

30. Karltorp E, Löfkvist U, Lewensohn-Fuchs I, et al. Impaired balance and neurodevelopmental disabilities among children with congenital cytomegalovirus infection. Acta Paediatr 2014;103:1165-73. 